\title{
Overcoming Barriers to Uptake of Voluntary Medical Male Circumcision in a Traditionally Circumcising Community in Machinga District, Malawi
}

\author{
Rodney J. Masese', Jane L. Chimango', Noel D. Mbirimtengerenji ${ }^{2}$ \\ ${ }^{1}$ Community and Mental Health Department, Kamuzu College of Nursing, University of Malawi, Lilongwe, Malawi \\ ${ }^{2}$ Medical-Surgical Nursing Department, Kamuzu College of Nursing, University of Malawi, Lilongwe, Malawi \\ Email: rmasese@kcn.unima.mw
}

How to cite this paper: Masese, R.J., Chimango, J.L. and Mbirimtengerenji, N.D. (2017) Overcoming Barriers to Uptake of Voluntary Medical Male Circumcision in a Traditionally Circumcising Community in Machinga District, Malawi. World Journal of AIDS, 7, 40-58.

https://doi.org/10.4236/wja.2017.71005

Received: January 3, 2017

Accepted: March 25, 2017

Published: March 28, 2017

Copyright $\odot 2017$ by authors and Scientific Research Publishing Inc. This work is licensed under the Creative Commons Attribution International License (CC BY 4.0).

http://creativecommons.org/licenses/by/4.0/

\begin{abstract}
Introduction: Voluntary medical male circumcision (VMMC) reduces chances of contracting HIV during heterosexual intercourse in males, and risk of cervical cancer in their female sexual partners. However, its uptake among traditionally circumcising communities, where male circumcision carries a cultural significance, has not been studied. Previous research has focused on barriers to uptake of VMMC in non-circumcising communities. This study was conducted to determine socio-cultural barriers to VMMC uptake in order to identify culture-sensitive and evidence-based interventions to increase its uptake. Methodology: This exploratory mixed methods cross-sectional study generated quantitative data from 262 randomly sampled sexually active men; and qualitative data through four focus-group discussions with 58 purposely selected male participants and 10 key informants. Results: The study established that only $6.3 \%(n=15)$ of those circumcised $(90.8 \%, n=238)$ were circumcised in clinical setting. Limited access to VMMC services, cost, pain, being attended to by female providers and cultural influences were the major hindrances to uptake of VMMC. Conclusion: Although there is a paradigm shift of preference from traditional male circumcision to VMMC in this community, its uptake is low. The study recommends that besides introducing mobile VMMC services, the Ministry of Health should collaborate with traditional circumcisers and local leaders to provide gender-and-culture-sensitive safer medical male circumcision services.
\end{abstract}

\section{Keywords}

VMMC, Medical Circumcision, Traditional Circumcision, Malawi, Uptake 


\section{Introduction}

Voluntary medical male circumcision (VMMC) has been proven to be a safe and effective strategy for reducing chances of acquiring HIV infection in males during heterosexual intercourse by approximately $60 \%$ [1] [2] [3]. This is particularly true if used in combination with other traditional strategies of HIV prevention, namely abstinence from unprotected sex, sticking to one faithful sexual partner, as well as correct and consistent condom use. Whilst male circumcision has been performed since recorded history for traditional as well as religious purposes, recently, the practice has globally been adopted as an HIV and AIDS preventive strategy [4] [5]. Studies have shown that the intervention not only prevents HIV infection in men, but also offers protection from cervical cancer in women whose partners are circumcised [6] [7].

In addition, VMMC is considered to be a significant life- and cost-saving intervention as it has been projected that up to two million new HIV infections and 300,000 AIDS-related deaths could be averted in the next 10 years in SubSaharan Africa [8] [9]. This is why the World Health Organisation (WHO) and the Joint United Nations Program on HIV and AIDS (UNAIDS) endorsed medical male circumcision as part of a comprehensive package for HIV prevention, mainly in high HIV prevalence and low male circumcision countries [10].

However, in spite of its proven efficacy, medical male circumcision is still perceived as a religious obligation practiced by some faith groups, particularly Muslims [6] [11]. As the World Health Organization (2009) estimates, $30 \%$ of all males older than 15 years, are circumcised globally. Nevertheless, out of this, only $16 \%$ are Christians, whilst $68 \%$ are Muslims [12] [13] [14].

Similarly, in Africa, the procedure is almost universal among the adult population in Muslim countries, but less common in the Southern African countries, where the majority is Christians [15]. Although there is dearth of statistics on medical circumcision, its prevalence is even lower in this region.

In Malawi, the national prevalence of male circumcision among the age group 15 - 49 years is estimated at $22 \%$ [16] [17]. Accordingly, the prevalence of medical circumcision is even much lower since according to World Health Organisation (WHO) standard definition, most of the circumcisions performed are traditional. This is because, historically, male circumcision was introduced in the context of religion and culture. As a result, its practice is generally viewed as an Islamic and ethnic ritual widely practiced by Muslims and traditionally circumcising Yao and Lhomwe tribes in Malawi [18]. Consequently, the general understanding of health benefits of VMMC, including prevention of HIV acquisition is very low.

Although modeling studies have shown that male circumcision could reduce HIV prevalence in southern Africa by up to 67\%, the actual impact on the epidemic depends upon uptake of voluntary medical male circumcision [2] [19] Thus, for new HIV infections to be averted, at least $80 \%$ of men aged $15-49$ years must be medically circumcised [20]. Hence, the central issue is how to enhance uptake of VMMC, particularly in traditionally circumcising communities, 
where male circumcision not only carries a considerable cultural meaning but also poses a greater risk for HIV transmission because of the unsafe environments in which male circumcisions are performed. This is probably why in Malawi the relationship between HIV prevalence and circumcision status is not in the expected direction as circumcised men have a higher HIV prevalence than those who have not been circumcised. For instance, with an HIV prevalence of $11 \%$ in the general population, it is $26 \%$ among circumcised men aged $35-44$ years [17].

Therefore, this study aimed at exploring barriers to uptake of VMMC among traditionally circumcising males in Machinga district in order to inform health policy makers on how to successfully scale up VMMC services in such areas. Traditional male circumcisions are widely known to increase the risk of contracting HIV and other sexually transmitted infections because of the unhygienic environments in which circumcisions are performed. The study findings will therefore assist policy makers in the Ministry of Health to develop evidencebased policies and strategies for efficient and effective implementation of VMMC services in such traditionally circumcising communities. Accordingly, the third sustainable development goal of ensuring healthy lives and ending the AIDS epidemic by 2030 will be realised.

\section{Methods}

\subsection{Study Design}

The study used a cross-sectional design which was exploratory in nature, triangulating quantitative and qualitative methods of data collection and analysis. This study was conducted in Machinga, one of the traditionally circumcising districts in the southern region of Malawi. Quantitative data and qualitative focus group discussion data were collected concurrently by trained indigenous male field interviewers to elicit attitudes and perceptions which would not have been gathered utilising quantitative methods alone. The field interviewers were Health Surveillance Assistants (HSAs) who were trained before collecting data in order to familiarise themselves with the data collection instrument and the actual exercise.

\subsection{Sampling Criteria and Sample Size}

In order to achieve a sample worthy generalisation, quantitatively 262 males aged between 15 - 49 years were randomly recruited from the target population. This was a sample size based on the single cross-sectional survey sample calculation as recommended by Gorstein, Sullivan, Parvanta and Begin (2007) [21].

The participants were located in their homes, where every third household in the randomly selected villages in Traditional Authority Chamba was picked to identify a potential respondent. Interviews were carried out in private convenient locations at participants' homes as proposed by them. Only sexually active males aged between 15 - 49 years who were permanent residents and were capable of answering questions about medical circumcision and sexual activity were 
recruited for the study.

\subsection{Ethical Considerations}

The study protocol and informed consent process were reviewed and approved by the University of Malawi (UNIMA) through the College of Medicine Research and Ethics Committee (COMREC). Local administrative officials and traditional leaders also gave approval for the study to be conducted in their area.

\subsection{Data Collection and Management}

Quantitative and qualitative data were collected concurrently between $7^{\text {th }}$ January and $20^{\text {th }}$ February, 2014 by trained indigenous field interviewers using guides. The interview and focus group guides were pretested amongst two focus group discussions and four in-depth interviews in a neighbouring village following two days of training. Prior to data collection, an initial visit was made to district and community authorities in order to explain the rationale and objectives of the survey. The Traditional Authority, Group Village Headmen and Village Headmen for the area were approached to seek their permission and sensitise their subjects in readiness for the study.

Individual interviews, which took 20 - 30 minutes, were carried out in private convenient locations at participants' homes as proposed by them. Focus groups were conducted at the Traditional Authority's court room, which was a central and private venue in the community. Key informants' interviews were also held at private and confidential venues in or near participants' homes. All the traditional circumcisers were interviewed in their duty rooms. On average, in-depth interviews and focus groups lasted 65 and 75 minutes, respectively.

Since the study was carried out in a Yao-speaking area, the research assistants were also from the same ethnic and age group as the participants in order to reduce social acceptability bias. The indigenous research assistants also led the focus group discussions and helped the principal investigator with translation. All discussions were digitally and manually recorded, and were then transcribed verbatim, and translated into English.

The questionnaires were coded and entered into the computer. Data cleaning was performed to check for errors in data entry prior to analysis.

\subsection{Data Analysis}

The quantitative data were analysed using Statistical Package for the Social Sciences (SPSS) version 16. Association between demographic variables, beliefs, and attitudes about medical circumcision, and decision making regarding voluntary medical male circumcision uptake was computed using Spearman's correlation. The test level of significance was $5 \%$.

Qualitative data were analysed through content analysis of identified themes. Data collected through interviews and focus groups were analysed iteratively. Themes were developed based on intuitioning, immersion, repeated reading, sorting, and coding of the data. The study used four criteria for establishing the 
trustworthiness of qualitative data collected from key informants and focus groups. These were credibility, dependability, conformability, and transferability. Bias was reduced through face to face interview during data collection.

\section{Results}

\subsection{Study Participants' Characteristics}

The socio-demographic characteristics and the self-reported circumcision status of the 262 survey respondents are summarized in Table 1 . Nearly half $(42 \%, \mathrm{n}=$ 110) of the interviewees were young males aged between 15 and 24 years. The

Table 1. Relationship of demographic variables and male circumcision status $(n=262)$.

\begin{tabular}{|c|c|c|c|c|}
\hline Item & Circumcised (\%) & Uncircumcised (\%) & Total (\%) & P-value \\
\hline Age group of respondents & & & & 0.003 \\
\hline $15-24$ years & $109(45.8)$ & $1(4.2)$ & $110(42.0)$ & \\
\hline $25-34$ years & $53(22.3)$ & $1(4.2)$ & $54(20.6)$ & \\
\hline 35 - 44 years & $36(15.1)$ & $6(25)$ & $42(16.0)$ & \\
\hline 45 - 49 years & $40(16.8)$ & $16(66.6)$ & $56(21.4)$ & \\
\hline Educational level & & & & 0.000 \\
\hline Primary & $152(63.9)$ & $12(50.0)$ & $164(62.6)$ & \\
\hline Secondary & $57(23.9)$ & $2(8.3)$ & $55(22.5)$ & \\
\hline None & $29(12.2)$ & $10(41.7)$ & $39(14.9)$ & \\
\hline Occupation & & & & 0.021 \\
\hline Schooling & 47 (19.7) & $1(4.2)$ & $48(18.3)$ & \\
\hline Farmer & $85(35.7)$ & $15(62.5)$ & $100(38.2)$ & \\
\hline Casual labourer & $65(27.3)$ & $2(8.3)$ & $67(25.6)$ & \\
\hline Employed & $3(1.3)$ & $1(4.2)$ & $4(1.5)$ & \\
\hline Business & $38(16.0)$ & $5(20.8)$ & $43(16.4)$ & \\
\hline Religion & & & & 0.000 \\
\hline Christian & $48(20.2)$ & $20(83.3)$ & $68(26.0)$ & \\
\hline Muslim & $190(79.8)$ & $4(16.7)$ & $194(74.0)$ & \\
\hline Tribe & & & & 0.000 \\
\hline Chewa & $17(7.1)$ & $2(8.3)$ & $19(7.3)$ & \\
\hline Yao & $188(79.0)$ & $6(25.0)$ & $194(74.0)$ & \\
\hline Lhomwe & $29(12.2)$ & $9(37.5)$ & $38(14.5)$ & \\
\hline Marital status & & & & 0.000 \\
\hline Single & $130(54.6)$ & $17(70.8)$ & $147(56.1)$ & \\
\hline Married & $94(39.5)$ & $1(4.2)$ & $95(36.3)$ & \\
\hline Widowed & $5(2.1)$ & $2(8.3)$ & $7(2.7)$ & \\
\hline Divorced & $9(3.8)$ & $4(16.7)$ & $13(5.0)$ & \\
\hline
\end{tabular}


rest were adults aged 25 years and above; with a mean age of 30.35 years. Similarly, $41.4 \%(n=24)$ of the participants in the focus group discussions were young. The majority $(90.8 \%, \mathrm{n}=238)$ of the interviewees reported to be circumcised at the time of the interviews. Two thirds $(66.6 \%, \mathrm{n}=16)$ of those uncircumcised were adults aged 45 - 49 years, implying that VMMC was particularly popular among the younger generation.

In terms of education, greater proportion $(62.6 \%, \mathrm{n}=164)$ of the participants attained primary education; $22.5 \%(\mathrm{n}=55)$ had secondary school education; whilst $14.9 \%(\mathrm{n}=39)$ had no education at all. As for occupation, over one third $(38.2 \%, \mathrm{n}=100)$ of the participants were subsistence farmers; whilst only $1.5 \%$ $(\mathrm{n}=4)$ were in formal employment.

All the participants were associated with some religion, the majority $(74 \%, \mathrm{n}=$ 194) being Muslims, and the remaining were Christians. The majority $(74 \%, \mathrm{n}=$ 194) of the respondents were Yao by tribe; whilst $14.5 \%(\mathrm{n}=38)$ were Lhomwe. With regard to marital status, more than half of the participants $(56.1 \%, \mathrm{n}=147)$ reported to be unmarried; whilst $35.9 \%(\mathrm{n}=94)$ were married and the remaining $8 \%$ were either divorced or widowed. As shown in Table 1, almost all the demographic variables were related to male circumcision since their $\mathrm{P}$-values were $\mathrm{P}<$ 0.05 . However, there was no relationship between occupation and circumcision status $(\mathrm{P}>0.05)$.

\subsection{Uptake of VMMC}

Out of the participants who were circumcised $(n=235)$, only $6.3 \%(n=15)$ were circumcised in clinical setting, the majority had undergone traditional circumcision. Over $50 \%(\mathrm{n}=8)$ of those who had undergone VMMC were young men aged 15 - 24 years and had attained primary education $(73.3 \%, \mathrm{n}=11)$. As shown in Table 2, there was significant negative correlation between age of respondents and uptake of VMMC $\left(\chi^{2}=54.531, \mathrm{df}=32, \mathrm{p}=0.008\right)$. It was shown that as people grow older, the uptake of VMMC goes down.

There was also significant association between marital status of respondents and medical circumcision $\left(\chi^{2}=11.811, \mathrm{df}=3, \mathrm{p}=0.008\right)$, with the uptake of VMMC being higher among unmarried males as nearly half $(46.7 \%, \mathrm{n}=7)$ of the respondents were unmarried. However, there was no significant correlation between VMMC uptake and other socio-demographic data.

\subsection{Perceived Barriers to VMMC Uptake}

Participants in both focus group discussions and during interviews highlighted important barriers to VMMC uptake. The qualitative findings on why men still opt for traditional circumcision despite social marketing of VMMC were consistent with the quantitative survey results. As summarised in Table 3, the most prominent barriers to VMMC among all survey respondents are accessibility (37\%), cost (27\%), culture (19\%), fear of pain (63\%), and bleeding (31\%). However, during FGDs, participants explained that presence of female providers in the circumcision team deters some men from accessing VMMC services. 
Table 2. Relationship of demographic characteristics and VMMC uptake.

\begin{tabular}{|c|c|c|c|c|}
\hline Characteristic & Traditional MC (\%) & $\operatorname{VMMC}(\%)$ & Total (\%) & $P$-value \\
\hline Age group of respondents & & & & 0.008 \\
\hline $15-24$ years & $101(45.4)$ & $8(53.6)$ & $109(45.9)$ & \\
\hline $25-34$ years & $52(23.4)$ & $1(6.7)$ & $53(22.3)$ & \\
\hline $35-44$ years & $35(15.6)$ & $1(6.7)$ & $36(15.2)$ & \\
\hline $45-49$ years & $35(15.6)$ & $5(33)$ & $40(16.6)$ & \\
\hline Education & & & & 0.329 \\
\hline None & $29(13.0)$ & $0(0.0)$ & $29(12.2)$ & \\
\hline Secondary & $53(23.8)$ & $4(26.7)$ & $57(23.9)$ & \\
\hline Primary & $141(63.2)$ & $11(73.3)$ & $152(63.9)$ & \\
\hline Occupation & & & & 0.953 \\
\hline Business & $36(16.1)$ & $2(13.3)$ & $38(16.0)$ & \\
\hline Employee & $3(1.3)$ & $0(0.0)$ & $3(1.3)$ & \\
\hline Casual labourer & $61(27.4)$ & $4(26.7)$ & $65(27.3)$ & \\
\hline Farmer & $80(35.9)$ & $5(33.3)$ & $85(35.7)$ & \\
\hline Schooling & $43(19.3)$ & $4(26.7)$ & $47(19.7)$ & \\
\hline Religion & & & & 0.987 \\
\hline Muslim & $178(79.8)$ & $12(80)$ & $190(79.8)$ & \\
\hline Christian & $45(20.2)$ & $3(20)$ & $48(20.2)$ & \\
\hline Tribe & & & & 0.298 \\
\hline Tumbuka & $1(0.4)$ & $0(0.0)$ & $1(0.4)$ & \\
\hline Ngoni & $2(0.9)$ & $1(6.7)$ & $3(1.3)$ & \\
\hline Lhomwe & $26(11.7)$ & $3(20)$ & $29(12.2)$ & \\
\hline Yao & $178(79.7)$ & $10(66.6)$ & $188(79.0)$ & \\
\hline Chewa & $16(7.2)$ & $1(6.7)$ & $17(7.1)$ & \\
\hline Marital status & & & & 0.008 \\
\hline Divorced & $6(2.7)$ & $3(20)$ & $9(3.8)$ & \\
\hline Widowed & $5(2.2)$ & $0(0.0)$ & $5(2.1)$ & \\
\hline Married & 89 (39.9) & $5(33.3)$ & $94(39.5)$ & \\
\hline Single & $123(55.2)$ & $7(46.7)$ & $130(54.6)$ & \\
\hline
\end{tabular}


Table 3. Factors hindering uptake of VMMC $(n=262)$.

\begin{tabular}{ccc}
\hline Item & Value & Percentage (\%) \\
\hline Hindrances for VMMC & 8 & 3.1 \\
Religion & 50 & 19.0 \\
Culture & 6 & 2.4 \\
Fear complication & 9 & 3.4 \\
No interest & 13 & 5.0 \\
Peer opposition & 97 & 37.0 \\
Accessibility & 71 & 27.0 \\
Cost & 8 & 3.1 \\
Misconceptions & & \\
\hline Negative consequences of VMMC & 10 & 3.8 \\
\hline Infections & 5 & 1.9 \\
Impotence & 82 & 31.1 \\
Bleeding & 165 & 63.0 \\
Pain & 5 & \\
\hline
\end{tabular}

Open-ended questions where participant may give none, one, or more than one response therefore proportion in table may not add up to $100 \%$.

\subsection{Cost and Accessibility}

Both components of data identified access to VMMC and cost as major hindrances to uptake of VMMC. Many participants stated that the cost incurred in form of either transport to access free VMMC services or payment for the procedure in private health facilities was beyond their means. One participant said "Many people here are too poor to afford MK400 transport money, and MK5, 000 to pay for medical circumcision services. As such, boys rush to traditional circumcision camps to run away from being left alone uncircumcised when all their peers are circumcised" (PT 10).

Nevertheless, some participants argued that traditional circumcision was in fact more expensive, considering the cost of payment for food for the initiates for a period of not less than a month, and many other requirements that go into the ceremony, including feasting. This, they said, contrasts with getting circumcised at the hospital which is less costly since one may spend just a day or two before being discharged home.

\subsection{Cultural Influence}

Culture was identified as another contributing factor to the lower uptake of VMMC by $19 \%(\mathrm{n}=50)$ of the interviewees who indicated that, culturally males are supposed to go for traditional circumcision. Similar sentiments were aired by some of the FGD participants who explained that male circumcision in the study area was regarded as an influential cultural practice where young males are 
counseled on how to behave and respect elders. While some men preferred medical circumcision, women were particularly in support of traditional circumcision for their sons. For instance, one participant in the older men group explained, "Women support each other financially through loans which are repaid with interests during initiation ceremonies, like business. If a man insists that my son will undergo medical circumcision, you will quarrel until you fight; even the marriage will break" (PT 4).

In addition, some participants explained that both chiefs and traditional circumcisers accrue financial gains from traditional circumcision, and therefore would hardly promote VMMC. One participant in the FGD stated, "Chiefs were a hindrance in promoting medical circumcision since they get money for each boy who goes for traditional circumcision. Because of such a reward it was difficult for local leaders to advocate for medical circumcision" (PT 9).

\subsection{Lack of Secrecy and Presence of Female Providers}

The most striking finding expressed by many participants in the younger focus group discussion was the hospitals' failure to purposely provide a secretive and all-male environment reminiscent of traditional male circumcision setting. Participants explained that such a lack of privacy creates an embarrassing atmosphere as women just pass by men who had been circumcised and are not kept in private rooms.

Whilst some participants expressed concern about the presence of female health providers in the operating room as compromising the privacy and secrecy characteristic of male circumcision, others did not mind. For instance, one participant stated "At the hospital you find that female providers are performing circumcisions; so some feel embarrassed thinking that "if she touches my private parts I will erect since I'm not sick. It is better in the village because I will be circumcised by my fellow men" (PT 7). On the other hand, one participant countered, "Whether you are attended to by a male or a female provider it does not matter, even female clients are sometimes cared for by male providers, for example during labour". However, those who opposed the involvement of female providers counter argued that unlike labouring women who would not mind being attended to by male providers because of intense pain, males are circumcised whilst in good health with all their senses intact.

\subsection{Fear of Pain and Bleeding}

Some participants indicated that fear of complications associated with VMMC kept away some men from undergoing the procedure, indicating that it was an operation that involves cutting off the foreskin. Fear of pain associated with undergoing circumcision was a concern stated quite often $(60 \%)$, whilst $31 \%$ of the interviewees identified bleeding as another factor preventing men from accessing VMMC services (Table 3). Participants' fear for pain was attributed to injection given before the procedure, cutting off of the foreskin, and post-operative healing. This was highlighted by one participant, "The procedure is very painful 
even though you are given an injection to relieve the pain. After you are operated on, you can not wear your underwear, but chitenje (female cloth). Even when walking women would know that you are injured. In rare cases one can die of severe bleeding" (PT 11).

However, some participants argued that less pain is experienced when one is circumcised at the hospital because "you are given good treatment which makes you not to experience intense pain; wound healing is also faster" (PT 1).

\section{Discussion}

The study was designed to identify factors hindering uptake of VMMC in a traditionally circumcising community in Machinga district in Malawi, and to generate evidence-based interventions to address these obstacles. Previous studies have focused on uptake of VMMC in non-circumcising communities, mostly using quantitative methods. This study used mixed methods and targeted men as well as traditional leaders and circumcisers to unearth socio-economic, cultural, and physical factors hindering VMMC uptake in order to develop successful interventions for addressing barriers to VMMC uptake.

\subsection{Respondents' Characteristics}

The study population is a sample of mostly Muslim Yao (74\%), young ( $42 \%$ aged between 15 - 24 years), unmarried (56\%), and traditionally-circumcised (90\%). Further analysis of both components of data established that most of those circumcised were sexually active youths aged between 15 and 24 years. Additionally, many were circumcised whilst young, with nearly two thirds circumcised at a mean age of 10 years. This suggests that age was strongly associated with circumcision status since many of those circumcised were the youth. Many studies concur that male circumcisions are commonly performed whilst young, as a rite of passage from childhood to adulthood, before initiation of sexual activity for quicker wound healing [22] [23] [24] [25] [26].

Although the 2010 Malawi Demographic Health Survey also found that majority of males $(87 \%)$ in this predominantly Muslim district were circumcised between the ages of 0 - 15 years, male circumcision prevalence rate was $85.4 \%$ [17]. The modest increase in the prevalence (90\%) of male circumcision in the current study could be attributed to the widespread campaign for adoption of voluntary medical male circumcision by both health workers and males from other tribes or cultures that do not traditionally circumcise.

In addition, there was no confirmation of actual circumcision status since the study did not include any physical examination on respondents who were reportedly circumcised. Without such confirmation, a false rate of circumcision could have been registered as some men could report that they were circumcised when in actual fact they were not. Such discrepancies were also noted in an operational study of uptake of medical male circumcision in a South African community where almost half of self-reported circumcised men had in fact an intact foreskin [8]. 


\subsection{Barriers to VMMC Uptake: Cost and Accessibility, Female Circumcisers, Culture, Fear of Pain and Bleeding}

The uptake of VMMC in the study area was only $6.3 \%$. In contrast, two separate studies carried out to determine uptake of adult male circumcision for HIV prevention in South African communities found VMMC uptake of 33\% [27] and $58.8 \%$ [8]. The key barriers to VMMC uptake that emerged from both the quantitative and qualitative data in the current study were: cost and accessibility, presence of female circumcisers, cultural influence, fear of pain and bleeding.

Lack of funds to access VMMC services for free, either at a far away government hospital or payment for the procedure at a nearby Mission hospital was a major barrier in the study area, as is also the case in Zambia and Kenya [2] [28]. Participants indicated that males who did not have enough money for either transport or payment for VMMC failed to access the service since centres offering the service for free were located far away. Typically, rural areas in Malawi are associated with poor infrastructure, inaccessible road network, and long distances to health facilities. It is these long distances, coupled with a lack of money which hinders men from accessing medical circumcision services. This finding is congruent with a study on acceptability of medical circumcision in a traditionally circumcising community in Tanzania, where cost for medical male circumcision procedure was a barrier, particularly among poor rural communities who were less likely to pay for medical circumcision than in traditional setting [29].

Long distance and lack of money for transport to access reproductive health services have also been acknowledged as the major concerns for people living in the rural areas in Malawi [30]. This is why, in order to conform to their circumcised peers, young males rush for traditional circumcision as an alternative since service payment is negotiable. A similar study in Uganda found that lack of nearby health centers offering VMMC and increased cost force many males to resort to traditional circumcisers who accept non-monetary payment [31]. For this reason, participants recommended that health authorities should increase access to safe VMMC services by making sure that free services are routinely provided at rural health centers so that men could access services closer to their homes.

Alternatively, participants recommended that traditional male circumcisers be trained and integrated into the national health delivery system as proposed elsewhere [32]. This would not only ensure that traditional circumcisers are supported and supervised by the district health care team, but also enhance safety in the traditional settings.

\subsection{Lack of Secrecy and Presence of Female Providers}

The study found the hospital environment to be a source of humiliation because of lack of privacy as well as the presence of female providers. Many older participants pointed out that lack of special rooms to keep circumcised men out of sight of relatives and other villagers who visit the hospital was perceived shameful. Such an environment compromises the status of adult men who enjoy a pri- 
vileged place in society since it reveals the fact that they were uncircumcised [33]. This is particularly true considering the fact that the majority of men are from a background of traditional circumcision, which is performed in secrecy. These findings correspond to those of other studies which observed that being too old was the main reason that made men to be reluctant to undergo VMMC [34] [35] [36].

Similarly, many older participants expressed that being attended to by female health providers was embarrassing, particularly considering the fact that one was not sick. The major reason given was that some men would erect as a female provider prepares them for circumcision. In fact, male circumcision was valued as a sensitive issue carried out in a male-only setting in many traditionally circumcising communities. This finding concurs with others which found that many men are not comfortable with women clinicians being part of the circumcising team for fear of being exposed as men who had not been circumcised, and the potential for sexual arousal [37] [38].

The implication of this finding is that health workers need to be more sensitive to the needs of men, particularly in such traditionally-circumcising communities if the uptake of VMMC is to be increased. This would include respecting social and cultural values, besides upholding ethical principles of privacy and confidentiality. Wherever possible, male health providers should perform VMMC especially for those clients who are very particular about sex differences of health providers.

\subsection{Fear of Pain and Bleeding}

Except for bleeding, fear of pain during the circumcision procedure and the recovery period was the most common perceived barrier to uptake of VMMC in this study, and is also regularly cited as a hindrance in other studies in Zambia, Zimbabwe, and Uganda [28] [39] [40]. In contrast, fear of pain during and after the procedure was not perceived as a major barrier to VMMC in some traditionally circumcising communities [41] [42], although it was so in other settings [2] [43]. Even in a recent medical male circumcision campaign, only a very small proportion (less than 1\%) of adverse events was reported [44]. Therefore, the misconception about complications associated with VMMC in the current study could be associated with inadequate knowledge about VMMC, previous experiences with complications commonly encountered with traditional circumcision, or misinformation from other people.

This finding on exaggerated apprehension of pain as a result of rumours and stories has also been noted in another study [2]. Such misconceptions from colleagues who share their traumatic experiences during traditional circumcision would deter some males who intend to undergo VMMC. Nevertheless, this underscores the need to uphold quality control standards in order to minimise pain, prevalence of post operative complications and other adverse outcomes. It also necessitates scaling up of information, education, and communication regarding safety of VMMC. This would then attract more males to undergo VMMC 
because of its perceived utmost safety.

\subsection{Cultural Influence}

Both survey respondents and some FGD participants indicated that male circumcision in the area was an influential cultural practice for graduating young males into adulthood. As with many societies that practice traditional circumcision, initiates are kept in seclusion where they are given instruction in cultural responsibilities, self-discipline, and general good behaviours [45] [46]. Whilst some women persuaded their sons to undergo traditional circumcision for socialisation, the study found that financial rewards to both traditional circumcisers (Angaliba) and traditional leaders motivate its continued practice. This finding is consistent with a study on prevalence and complications of traditional male circumcision in East and Southern Africa which found that some traditional circumcisers just seize the opportunity to earn money without necessarily undergoing formal training for safe circumcision [38]. Sadly, this predisposes young initiates to HIV infection and other life-threatening complications since such circumcisions are generally performed under unhygienic conditions and without use of anaesthesia [23] [47] [48].

Whilst previous studies found women in traditionally circumcising communities to be supportive of medical male circumcision for their sons [29] [49], this study found that women preferred their children to undergo traditional male circumcision for its perceived cultural benefits. A similar study found that traditional circumcisions are valued as an important stage of initiation for boys from infancy into adulthood [50]. This is why the young boys had a perception of social pressure to comply with the decision of their mothers who are their important referents [51]. The implication of this is that among the young boys, their mothers' opinions regarding VMMC remains important. Therefore, women as referents of their sons need to be targeted with accurate information so that their attitude and perception regarding VMMC are positive. This would transform the mindset of the women to perceive VMMC as more of a health promotion intervention than merely a cultural phenomenon.

On the other hand, the study found that chiefs' pivotal role of changing the perception of their subjects and addressing cultural barriers to VMMC uptake was not well executed. As pointed out by study participants, local leaders can influence an end to the practice of traditional circumcision. Although some studies argue that clinical male circumcision will never completely replace traditional practices due to the cultural implications and the human resource constraints [29] [38] [52], local leaders need to spearhead the change. Not only do chiefs have the powers to alter the practice of traditional circumcision, but can also persuade men to get circumcised in clinical setting where safety is guaranteed. In fact, traditional leadership and support for medical circumcision completely changes the location of male circumcision from traditional to clinical settings [49]. Therefore, targeting chiefs as custodians of culture, as well as traditional circumcisers and religious leaders with accurate messages regarding health bene- 
fits of VMMC and dangers of traditional circumcision would help to motivate all males to undergo VMMC.

More importantly, working with all stakeholders such as local leaders and traditional circumcisers as well as religious leaders as recommended by one study [25] is imperative since religion determines where and when male circumcision should occur [53]. One important paradigm shift is to encourage young males to get circumcised in the clinical setting, and continue with the other harmless traditional activities like cultural festive. This would greatly reduce the practice of traditional circumcision in the study area and therefore increase VMMC uptake.

The study makes several recommendations. Firstly, Ministry of Health officials should engender collaboration with traditional circumcisers to empower them and carry out new roles. This would include motivating and referring males for culture-sensitive mobile VMMC services within their locality. In addition, the VMMC national program should scale up innovative behavioural change communication strategies to motivate more young males to undergo VMMC. More importantly, religious and local leaders should be targeted with accurate messages regarding the public health benefits of VMMC for them to change their attitude and motivate their subjects.

\section{Study Limitations}

Findings from this study should be interpreted in light of its limitations in generalisability to the larger population since the sample was restricted to a specific population group in one traditionally circumcising district. Also, representativeness of VMMC clients could not be independently verified since circumcision status of respondents relied on self-reported data. The other limitation is that views of women regarding their support for VMMC for their sons were not registered since the study only targeted males. Whereas men in this study stated that women were less supportive of VMMC for their sons, it was imperative to get the views of the women themselves. However, the sampling strategy and use of mixed methods in data collection and analysis compensated for most of these study limitations. As such, the study still provides important insight regarding uptake of VMMC in traditionally circumcising communities in Malawi.

\section{Conclusion}

This mixed methods study has unearthed significant insight into practical barriers to the uptake of VMMC in a traditionally circumcising community in Malawi. Although there is a paradigm shift of preference from traditional male circumcision to VMMC in this community, its uptake is only $6.3 \%$. The findings from both quantitative and qualitative data sources recognise long distances in accessing free VMMC services, provision of services by female health workers, cultural influence, and fear of pain as the major barriers to uptake of VMMC. Nevertheless, study participants provided possible strategies to overcome the challenges and ultimately increase access to medical circumcision. These findings can inform the national VMMC program to collaborate with traditional 
leaders and circumcisers to enhance uptake of VMMC. However, additional research is needed to understand the role of women and traditional circumcisers on VMMC uptake in traditionally circumcising communities.

\section{Acknowledgements}

This study was made possible by the generosity of several players, whom the authors would like to recognise. Local leaders in the study area need to be commended for their acceptance and support to have the study carried out. We would also like to thank the study participants who kindly provided their time for us to gain insights into some challenges rocking the VMMC program and how to overcome them.

\section{Competing Interests}

The authors declare that they have no competing interests.

\section{References}

[1] Auvert, B., Taljaard, D., Lagarde, E., Sobngwi-Tambekou, J., Sitta, R. and Puren, A. (2005) Randomized, Controlled Intervention Trial of Male Circumcision for Reduction of HIV Infection Risk: The ANRS 1265 Trial. PLoS Medicine, 2, e298. https://doi.org/10.1371/journal.pmed.0020298

[2] Bailey, R.C., Moses, S., Parker, C.B., Agot, K., Maclean, I., Krieger, J.N. and NdinyaAchola, J.O. (2007) Male Circumcision for HIV Prevention in Young Men in Kisumu, Kenya: A Randomised Controlled Trial. The Lancet, 369, 643-656. https://doi.org/10.1016/S0140-6736(07)60312-2

[3] Gray, R.H., Kigozi, G., Serwadda, D., Makumbi, F., Watya, S., Nalugoda, F., et al. (2007) Male Circumcision for HIV Prevention in Men in Rakai, Uganda: A Randomised Trial. The Lancet, 369, 657-666. https://doi.org/10.1016/S0140-6736(07)60313-4

[4] Aggleton, P. (2007) "Just a Snip"? A Social History of Male Circumcision. Reproductive Health Matters, 15, 15-21. https://doi.org/10.1016/S0968-8080(07)29303-6

[5] Auvert, B., Lissouba, P., Geffen, N., Fiamma, A. and Heywood, M. (2009) Key Facts on Male Circumcision. South African Medical Journal, 99, 150-151.

[6] Njeuhmeli, E., Forsythe, S., Reed, J., Opuni, M., Bollinger, L., Heard, N., et al. (2011) Voluntary Medical Male Circumcision: Modeling the Impact and Cost of Expanding Male Circumcision for HIV Prevention in Eastern and Southern Africa. PLoS Medicine, 8, e1001132. https://doi.org/10.1371/journal.pmed.1001132

[7] UNAIDS/WHO (2011) Joint Strategic Action Framework to Accelerate the ScaleUp of Voluntary Medical Male Circumcision for HIV Prevention in Eastern and Southern Africa.

[8] Lissouba, P., Taljaard, D., Rech, D., Dermaux-Msimang, V., Legeai, C., Lewis, D., et al. (2011) Adult Male Circumcision as an Intervention against HIV: An Operational Study of Uptake in a South African Community (ANRS 12126). BMC Infectious Diseases, 11, 253. https://doi.org/10.1186/1471-2334-11-253

[9] Williams, B.G., Lloyd-Smith, J.O., Gouw, E., Hankins, C., Getz, W.M., Hargrove, J., et al. (2006) The Potential Impact of Male Circumcision on HIV in Sub-Saharan Africa. PLoS Medicine, 3, e262. https://doi.org/10.1371/journal.pmed.0030262

[10] World Health Organization (2013) Use of Devices for Adult Male Circumcision in 
Public Health HIV Prevention Programmes. http://www.who.int/hiv/pub/malecircumcision/devices_conclusions/en/index.html

[11] Iliyasu, Z., Abubakar, I.S., Jibo, A.M. and Salihu, H.M. (2012) Male Circumcision and HIV Risk Behavior among University Students in Northern Nigeria. American Journal of Men's Health, 7, 94-101. https://doi.org/10.1177/1557988312456698

[12] World Health Organization (2009) Country Experience in the Scale-Up of Male Circumcision in Eastern and Southern Africa Region: Two Years and Counting. http://www.who.int/hiv/pub/malecircumcision/cntry_experiences_se_africa_06.09. 09.pdf?ua=1

[13] Drain, P.K., Daniel, D.T., Hughes, J.P., Klausner, J.D. and Bailey, R.C. (2006) Male Circumcision, Religion, and Infectious Diseases: An Ecologic Analysis of 118 Developing Countries. BMC Infectious Diseases, 6, 172. https://doi.org/10.1186/1471-2334-6-172

[14] Hankins, C. (2007) Male Circumcision: Implications for Women as Sexual Partners and Parents. Reproductive Health Matters, 15, 62-67. https://doi.org/10.1016/S0968-8080(07)29311-5

[15] Peltzer, K., Niang, C.I., Muula, A.S., Bowa, K., Okeke, L., Boiro, H. and Chimbwete, C. (2007) Editorial Review: Male Circumcision, Gender and HIV Prevention in Sub-Saharan Africa: A (Social Science) Research Agenda. Journal of Social Aspects of HIVI AIDS, 4, 658-667. https://doi.org/10.1080/17290376.2007.9724889

[16] Republic of Malawi (2009) National HIV Prevention Strategy 2009-2013. Ministry of Health, Lilongwe.

[17] National Statistical Office (2011) Malawi Demographic and Health Survey 2010. National Statistical Office, Zomba, Malawi.

[18] Bengo, J., Chalulu, K., Chinkhumba, J., Kazembe, L., Maleta, K., Masiye, F. and Mathanga, D. (2010) Situation Analysis of Male Circumcision in Malawi. College of Medicine, Blantyre.

[19] Nagelkerke, N., Moses, S., de Vlas, S. and Bailey, R. (2007) Modeling the Public Health Impact of Male Circumcision for HIV Prevention in High Prevalence Areas in Africa. BMC Infectious Diseases, 7, 16-30. https://doi.org/10.1186/1471-2334-7-16

[20] World Health Organization (2012) Voluntary Medical Male Circumcision for HIV Prevention. http://www.who.int/hiv/topics/malecircumcision/fact_sheet/en/index.html

[21] Gorstein, J., Sullivan, K.M., Parvanta, I. and Begin, F. (2007) Indicators and Methods for Cross-Sectional Surveys of Vitamin and Mineral Status of Populations. CDC and Micronutrient Initiative, Atlanta.

[22] Andersson, N. and Cockcroft, A. (2012) Male Circumcision, Attitudes to HIV Prevention and HIV Status: A Cross-Sectional Study in Botswana, Namibia and Swaziland. AIDS Care, 24, 301-309. https://doi.org/10.1080/09540121.2011.608793

[23] Meissner, O. and Buso, D.L. (2007) Traditional Male Circumcision in the Eastern Cape: Scourge or Blessing? South African Medical Journal, 97, 371-373.

[24] Nyembezi, A., Funani, I., Sifunda, S., Ruiter, R., van den Borne, B. and Reddy, P. (2012) The Psychosocial Determinants of the Intention to Reduce the Number of Sexual Partners among Recent Traditionally Initiated and Circumcised Men in the Eastern Cape Province, South Africa. Journal of Health Psychology, 17, 664-675. https://doi.org/10.1177/1359105311424469

[25] Herman-Roloff, A., Llewellyn, E., Obiero, W., Agot, K., Ndinya-Achola, J., Muraguri, N. and Bailey, R.C. (2011) Implementing Voluntary Medical Male Circumcision 
for HIV Prevention in Nyanza Province, Kenya: Lessons Learned during the First Year. PLoS ONE, 6, e18299. https://doi.org/10.1371/journal.pone.0018299

[26] Binagwaho, A., Pegurri, E., Muita, J. and Bertozzi, S. (2010) Male Circumcision at Different Ages in Rwanda: A Cost-Effectiveness Study. PLoS Medicine, 7, e1000211. https://doi.org/10.1371/journal.pmed.1000211

[27] de Bruyn, G., Martinson, N.A., Nkala, B.D., Tshabangu, N., Shilaluka, G., Kublin, J., et al. (2009) Uptake of Male Circumcision in an HIV Vaccine Efficacy Trial. Journal of Acquired Immune Deficiency Syndromes, 51, 108-110. https://doi.org/10.1097/QAI.0b013e3181a03622

[28] Lukobo, M. and Bailey, R.C. (2007) Acceptability of Male Circumcision for Prevention of HIV Infection in Zambia. AIDS Care, 19, 471-477. https://doi.org/10.1080/09540120601163250

[29] Wambura, M., Mwanga, J.R., Mosha, J.F., Mshana, G., Mosha, F. and Changalucha, J. (2011) Acceptability of Medical Male Circumcision in the Traditionally Circumcising Communities in Northern Tanzania. BMC Public Health, 11, 373. https://doi.org/10.1186/1471-2458-11-373

[30] Ministry of Health (2011) Malawi Health Sector Strategic Plan 2011-2016: Moving towards Equity and Quality. Ministry of Health, Lilongwe.

[31] Ramirez, C.B. and Bufumbo, L. (2010) Formative Research to Inform the Rollout of Medical Male Circumcision in Communities Where Traditional Circumcision Is Practiced in Uganda.

[32] Mwanga, J.R., Wambura, M., Mosha, J.F., Mshana, G., Mosha, F. and Changalucha, J. (2011) Policy Environment and Male Circumcision for HIV Prevention: Findings from a Situation Analysis Study in Tanzania. BMC Public Health, 11, 506. https://doi.org/10.1186/1471-2458-11-506

[33] Plotkin, M., Castor, D., Mziray, H., Küver, J., Mpuya, E., Luvanda, P. J., et al. (2013) “Man, What Took You So Long?" Social and Individual Factors Affecting Adult Attendance at Voluntary Medical Male Circumcision Services in Tanzania. Global Health: Science and Practice, 1, 108-116. https://doi.org/10.9745/GHSP-D-12-00037

[34] Gasasira, R.A., Sarker, M., Tsague, L., Nsanzimana, S., Gwiza, A., Mbabazi, J. and Mugwaneza, P. (2012) Determinants of Circumcision and Willingness to Be Circumcised by Rwandan Men, 2010. BMC Public Health, 12, 134.

https://doi.org/10.1186/1471-2458-12-134

[35] Tarimo, E.A., Francis, J.M., Kakoko, D., Munseri, P., Bakari, M. and Sandstrom, E. (2012) The Perceptions on Male Circumcision as a Preventive Measure against HIV Infection and Considerations in Scaling up of the Services: A Qualitative Study among Police Officers in Dar es Salaam, Tanzania. BMC Public Health, 12, 529. https://doi.org/10.1186/1471-2458-12-529

[36] Bertrand, J.T., Njeuhmeli, E., Forsythe, S., Mattison, S.K., Mahler, H. and Hankins, C.A. (2011) Voluntary Medical Male Circumcision: A Qualitative Study Exploring the Challenges of Costing Demand Creation in Eastern and Southern Africa. PLoS ONE, 6, e27562. https://doi.org/10.1371/journal.pone.0027562

[37] Umar, E., Mandalazi, P., Jere, D. and Muula, A. (2013) Should Female Health Providers Be Involved in Medical Male Circumcision? Narratives of Newly Circumcised Men in Malawi. Malawi Medical Journal, 25, 72-77.

[38] Wilcken, A., Keil, T. and Dick, B. (2010) Traditional Male Circumcision in Eastern and Southern Africa: A Systematic Review of Prevalence and Complications. Bulletin of the World Health Organization, 88, 907-914. https://doi.org/10.2471/BLT.09.072975 
[39] Halperin, D.T., Fritz, K., McFarland, W. and Woelk, G. (2005) Acceptability of Adult Male Circumcision for Sexually Transmitted Disease and HIV Prevention in Zimbabwe. Sexually Transmitted Diseases, 32, 238-239. https://doi.org/10.1097/01.olq.0000149782.47456.5b

[40] Albert, L.M., Akol, A., L’Engle, K., Tolley, E.E., Ramirez, C.B., Opio, A., et al. (2011) Acceptability of Male Circumcision for Prevention of HIV Infection among Men and Women in Uganda. AIDS Care, 23, 1578-1585.

https://doi.org/10.1080/09540121.2011.579939

[41] Ngalande, R.C., Levy, J., Kaponda, C.P.N. and Bailey, R.C. (2006) Acceptability of Male Circumcision for Prevention of HIV Infection in Malawi. AIDS and Behavior, 10, 377-385. https://doi.org/10.1007/s10461-006-9076-8

[42] Westercamp, M. and Bailey, R.C. (2007) Acceptability of Male Circumcision for Prevention of HIV/AIDS in Sub-Saharan Africa: A Review. AIDS and Behavior, 11, 341-355. https://doi.org/10.1007/s10461-006-9169-4

[43] Mattson, C.L., Bailey, R.C., Muga, R., Onyango, T. and Poulussen, R. (2005) Acceptability of Male Circumcision and Predictors of Circumcision Preference among Men and Women in Nyanza Province, Kenya. AIDS Care, 17, 182-194. https://doi.org/10.1080/09540120512331325671

[44] Mahler, H.R., Kileo, B., Curran, K., Plotkin, M., Adamu, T., Hellar, A., et al. (2011) Voluntary Medical Male Circumcision: Matching Demand and Supply with Quality and Efficiency in a High-Volume Campaign in Iringa Region, Tanzania. PLoS Medicine, 8, e1001131. https://doi.org/10.1371/journal.pmed.1001131

[45] Kioli, F.N., Were, A.R. and Onkware, K. (2012) Traditional Perspectives and Control Mechanisms of Adolescent Sexual Behavior in Kenya. International Journal of Sociology and Anthropology, 4, 1-7. https://doi.org/10.5897/IJSAX11.002

[46] WHO (2009) Traditional Male Circumcision among Young People: A Public Health Perspective in the Context of HIV Prevention. World Health Organization. Geneva.

[47] Ministry of Health (2012) National Policy on Voluntary Medical Male Circumcision. Ministry of Health, Lilongwe.

[48] Bailey, R.C., Egesah, O. and Rosenberg, S. (2008) Male Circumcision for HIV Prevention: A Prospective Study of Complications in Clinical and Traditional Settings in Bungoma, Kenya. Bulletin of World Health Organization, 86, 669-677. https://doi.org/10.2471/BLT.08.051482

[49] Siegler, A.J., Mbwambo, J.K. and DiClemente, R.J. (2012) Acceptability of Medical male Circumcision and Improved Instrument Sanitation among a Traditionally Circumcising Group in East Africa. AIDS and Behavior, 16, 1846-1852. https://doi.org/10.1007/s10461-012-0262-6

[50] Mshana, G., Wambura, M., Mwanga, J., Mosha, F. and Changalucha, J. (2011) Traditional Male Circumcision Practices among the Kurya of North-Eastern Tanzania and Implications for National Programs. AIDS Care, 23, 1111-1116. https://doi.org/10.1080/09540121.2011.554518

[51] Ajzen, I. (1991) The Theory of Planned Behavior. Organizational Behavior and Human Decision Processes, 50, 179-211.

[52] Peltzer, K. and Kanta, X. (2009) Medical Circumcision and Manhood Initiation Rituals in the Eastern Cape, South Africa: A Post Intervention Evaluation. Culture, Health \& Sexuality, 11, 83-97. https://doi.org/10.1080/13691050802389777

[53] Sawires, S.R., Dworkin, S.L., Fiamma, A., Peacock, D., Szekeres, G. and Coates, T.J. (2007) Male Circumcision and HIV/AIDS: Challenges and Opportunities. The Lancet, 369, 708-713. https://doi.org/10.1016/S0140-6736(07)60323-7 


\section{Abbreviations}

COMREC: College of Medicine Research and Ethics Committee; FGD: Focus group discussion; HIV: Human Immunodeficiency Virus; MOH: Ministry of NSO: National Statistical Office; Health; STI: Sexually transmitted infection; UNAIDS: Joint United Nations Program on HIV and AIDS; VMMC: Voluntary medical male circumcision; WHO: World Health Organisation.

Submit or recommend next manuscript to SCIRP and we will provide best service for you:

Accepting pre-submission inquiries through Email, Facebook, LinkedIn, Twitter, etc. A wide selection of journals (inclusive of 9 subjects, more than 200 journals)

Providing 24-hour high-quality service

User-friendly online submission system

Fair and swift peer-review system

Efficient typesetting and proofreading procedure

Display of the result of downloads and visits, as well as the number of cited articles

Maximum dissemination of your research work

Submit your manuscript at: http://papersubmission.scirp.org/

Or contact wja@scirp.org 